\title{
Integração de preços no mercado da soja nos estados do Mato Grosso, Paraná e Rio Grande do Sul
}

\author{
Juliana de Sales Silva ${ }^{1}$ \\ Carlos Otávio de Freitas ${ }^{2}$ \\ João Eustáquio de Lima $^{3}$
}

\begin{abstract}
Resumo
O objetivo deste estudo é investigar as relações de longo prazo do preço da soja produzida no Mato Grosso, Paraná e Rio Grande do Sul, com o intuito de testar a validade da Lei do Preço Único entre esses mercados. As séries históricas representam os logaritmos naturais dos preços da soja de janeiro de 2005 a dezembro de 2015. Para determinar a relação de integração entre as variáveis, foram aplicados testes de raiz unitária de Dickey-Fuller aumentado e Phillips-Perron, co-integração de Johansen, teste de causalidade de Granger, funções impulso resposta, decomposição da variância, e testes sobre parâmetros $\beta$ e $\alpha$ do vetor de co-integração. Testou-se ainda a hipótese de perfeita integração, com o intuito de verificar se a lei de preço único é verdadeira para esses mercados. Entre os resultados obtidos, verificou-se que variações no preço da soja no Paraná são transmitidas quase integralmente para o Mato Grosso e Rio Grande do Sul. Os coeficientes de correção de erro ainda apontaram que os preços da soja se ajustam rapidamente a desequilíbrios de curto prazo nos preços nacionais desta commodity. Além disso, foi identificado mercados perfeitamente integrados, dando suporte à validação da Lei de Preço Único no mercado de soja exportada.
\end{abstract}

Palavras-Chave: soja, co-integração, lei de preço único.

Integration of prices in the soybean market in Mato Grosso, Paraná and Rio Grande do Sul

\begin{abstract}
The aim of this study is to investigate the long-term relationships of soybeans prices in Mato Grosso, Parana and Rio Grande do Sul, in order to test the validity of the Single Price Law between these markets. The historical series represent the natural logarithms of soy prices of January 2005 to December 2015. To determine the relationship between the variables of integration, were applied Dickey Fuller and Phillips-Perron unit root tests, co-integration Johansen, Granger causality test, impulse response functions, decomposition of variance and tests on parameters $\beta$ and $\alpha$ of the

1 Professora Assistente A da Universidade Federal do Sul e Sudeste do Pará. E-mail: julianasalessil-

2 Professor Assistente Classe A da Universidade Federal Rural do Rio de Janeiro. E-mail: carlos.

3 Professor Titular da Universidade Federal de Viçosa. E-mail: jelima@ufv.br
\end{abstract} va@live.com freitas@ufv.br 
co-integration vector. It also tested the hypothesis of perfect integration, in order to verify that the Single Price Law is true for those markets. Among the results, it was found that variations in the price of soybeans in Parana are transmitted almost entirely to the Mato Grosso and Rio Grande do Sul. Error correction coefficients also indicated that soybean prices adjust quickly to short term disequilibrium in national prices of this commodity. Moreover, perfectly integrated markets was identified, supporting the validation of Single Price Law of the exported soybean market.

Keywords: soybens, co-integration, single price law.

Classificação JEL: C32, Q13.

\section{Introdução}

De acordo com FAOSTAT (2014), o mercado de soja, tanto na produção quanto na exportação, caracteriza-se por domínio de três países: Estados Unidos, Brasil e Argentina. Assim, o destaque do Brasil como um dos principais produtores e exportadores de soja em grão, farelo e óleo de soja do mundo é resultado êxito na produção de soja das últimas décadas. No Gráfico 1 pode-se perceber a evolução das exportações do complexo de soja do País, a qual apresenta uma tendência crescente na série histórica.

Gráfico 1 - Exportações brasileiras do complexo de soja 2003 - 2013.

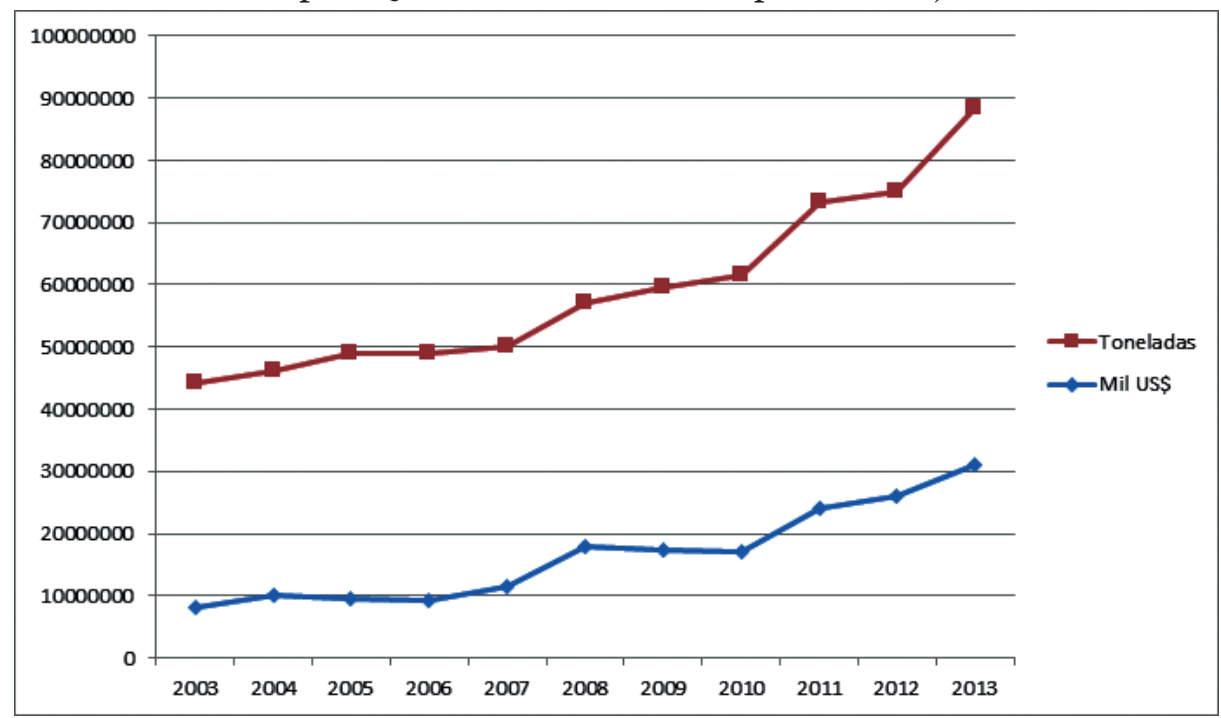

Fonte: Elaboração própria com dados BRASIL/MAPA, 2014. 
Conforme Silva et al. (2003) a soja é um dos produtos mais representativos na integração das atividades produtivas das cadeias agroindustriais do Brasil. De acordo com os autores, isto é, devido à participação do capital internacional que contribui com o desenvolvimento significativo do principal polo industrial desse complexo, sobretudo nos estados do Sul e Sudeste do País.

Ainda em relação ao mercado nacional de soja, os estados do Mato Grosso, Paraná e Rio Grande do Sul destacam-se como os principais produtores da commodity. De acordo com a Companhia nacional de abastecimento - CONAB (2016), a produção desses estados para a safra 2014/2015 foi, respectivamente, 28,0, 17,2 e 14,9 milhões de toneladas, representando um crescimento de $56,2 \%, 77,3 \%$ e $421 \%$, em relação à safra de 2004/2005. Além disso, em conjunto, os três estados foram responsáveis por aproximadamente $63 \%$ (60,1 milhões de ton.) da safra de soja no ano de 2014/2015.

Sousa e Campos (2009) destacam que análises de integração de mercados são importantes para o desenvolvimento econômico de um país, pois promovem as tomadas de decisão dos agentes envolvidos nos mercados e colaboram com a eficiência dos mesmos.

Diante disso, este trabalho busca investigar as relações de longo prazo do preço da soja exportada no Mato Grosso, Paraná e Rio Grande do Sul. Assim, verificar se a Lei do Preço Único (LPU) pode ser aplicada entre mercados de soja dos estados de Mato Grosso, Paraná e Rio Grande do Sul no período de janeiro de 2005 a dezembro de 2015 , isto é, se alterações de preços em um mercado poderão ser transmitidas para os demais.

Este artigo está dividido, além desta introdução, em quatro seções. Na primeira seção, têm-se uma breve revisão de literatura dos estudos sobre a transmissão e integração de preços; na segunda, a metodologia empregada neste estudo; na terceira, os resultados; e na última, as considerações finais.

\section{Revisão de Literatura}

Entre os trabalhos que abordam a transmissão e integração de preços, pode-se citar os de Lovadine (2004), Mattos (2008), Cunha (2008), Souza e Campos (2009), Christofoletti et al.(2011), Tardelli (2012), entre outros.

Lovadine (2004) busca identificar as relações causais e estimar as elasticidades de transmissão do preço internacional da soja no período de janeiro de 1999 a janeiro de 2004. A autora realiza testes de raiz unitária, co-integração de Engle e Granger e causalidade, chegando a conclusão de que há relações causais entre os preços interno e externo do farelo de soja e entre os preços interno e externo do óleo de soja. Quanto à causalidade, não foi identificada uma relação entre os preços interno e externo do grão de soja.

Mattos (2008) procura verificar, em seu trabalho, a transmissão de preços en- 
tre os mercados regionais de carne de frango no Brasil, se há diferenças nos custos de transações nos mercados. A técnica metodológica utilizada pelo autor é o Thresshold Cointegration e consta barreira à transmissão de preços entre os mercados, em função dos custos de transação. $O$ autor observa que, no curto prazo, não se espera co-integração entre os preços, que São Paulo é o estado formador de preços e há pouca sensibilidade a desvios na relação de equilíbrio de longo prazo. Quanto aos parâmetros de thresholds, a transmissão de preços é assimétrica.

Cunha (2008) investiga a integração de preços no mercado internacional do café. O autor utilizou o procedimento de Johansen e perfis de persistência e chegou à conclusão que Brasil, Colômbia, México, Guatemala, Peru e Honduras foram integrados entre si. Da mesma forma que os preços de café do Vietnã, Brasil e Indonésia. Assim, o mercado não é segmentado e existe transmissão de preços.

Sousa e Campos (2009) estimam as elasticidades de transmissão de preços da soja produzida no Mato Grosso, Rio Grande do Sul e Paraná, com o intuito de testar a validade da Lei do preço único entre esses mercados. Os autores concluem que as variações de preços de longo prazo, ocorridas no Rio Grande do Sul, são transmitidas quase que totalmente para os preços da soja no Paraná e no Mato Grosso, no entanto, a Lei do preço único não é perfeitamente verificada nesses mercados de soja.

Christofoletti et al.(2011) analisam o grau de integração e a relação de causalidade entre os mercados futuros da soja em grão de Brasil, EUA e China. Os autores observam que existe uma relação estrutural de equilíbrio de longo prazo entre as séries de preços analisadas. Quanto a causalidade, percebem a existência de bi-causalidade entre as séries de preços da soja nos mercados futuros do Brasil e da China.

Tardelli (2012) analisa o processo de integração espacial em mercados de soja do Mato Grosso com o porto de Santos considerando os custos de transação, para avaliar o grau de insuficiência da infraestrutura de escoamento entre as regiões produtoras e o porto de Santos. O autor utiliza o modelo TVEC e chega à conclusão que a grande extensão do estado de Mato Grosso interfere na transmissão de preços com o porto de Santos.

\section{Metodologia} trabalho.

Nesta seção serão apresentados os modelos teórico e analítico utilizados no

\subsection{Modelo teórico}

Integração de mercado é a extensão com que os choques são transmitidos de um mercado ao outro (GOODWIN; PIGGOTT, 2001). Cunha (2008) aponta que 
a principal variável na integração de mercado é o preço. Assim, os estudos de tal temática estão focados nas relações entre preços em diferentes localidades, que podem estar num mesmo país, ou em países, regiões ou blocos econômicos diferentes, avaliando o quanto estão relacionados.

Segundo Mattos (2008), a integração de mercado sempre esteve atrelada à interdependência de preços em distintos mercados, aludindo em conceitos de integração espacial e, sobretudo, na arbitragem espacial e Lei de Preço Único (LPU).

Conforme a LPU exposta por Krugman e Obstfeld (2005), os preços de bens idênticos, transacionados em mercados concorrenciais, sem custos de transação e barreiras às trocas, quando expressos na mesma moeda, não devem ser diferentes. A LPU, em sua forma mais simples, pode ser representada matematicamente da seguinte forma (FREITAS et al., 2014):

$$
\mathrm{P}_{\mathrm{it}}=\alpha+\beta \mathrm{P}_{\mathrm{jt}}+\mathrm{u}_{\mathrm{t}}
$$

em que, $P_{i t}$ e $P_{j t}$ são as representações logaritmizadas dos preços commodity (no presente trabalho, soja) nos estados i e j, no período t; $\alpha$ é a constante (ou intercepto); $\beta$ é a representação da elasticidade de transmissão de preço entre os estados analisados (Mato Grosso, Paraná e Rio Grande do Sul); e u é o termo de erro.

Quanto à elasticidade de transmissão de preço (parâmetro $\beta$ ), algumas hipóteses podem ser verificadas após a sua estimação. Caso $\beta$ seja igual à unidade, isto implica que variações de preço no mercado de soja em determinado estado serão plenamente transmitidas para o mercado de outro estado. Por outro lado, caso o valor de $\beta$ seja igual a zero, as variações de preço não serão transmitidas entre os estados.

Nesse sentido, se verificada a LPU, o preço de um produto tenderá a aumentar no mercado com menor preço, devido ao acréscimo da quantidade demandada, enquanto o preço tenderá a diminuir no mercado com maior preço, decorrente do excesso da quantidade ofertada, até que a diferença máxima seja o custo de transporte entre as regiões, isto é, até que os preços se igualem nos dois mercados. Essa relação é conhecida como condição de arbitragem espacial.

Conforme Fackler e Goodwin (2000) a Lei de preço único possui duas versões, uma fraca (2) e uma forte (3), sendo esta última a mais testada em estudos de integração de mercados de acordo com Mattos (2008).

$$
\begin{aligned}
& P_{i}-P_{j} \leq C_{j i} \\
& P_{i}-P_{j}=C_{j i}
\end{aligned}
$$

em que $\mathrm{P}_{\mathrm{i}}$ é o preço no mercado i, $\mathrm{P}_{\mathrm{j}}$ é o preço no mercado $j$, e $\mathrm{C}_{\mathrm{ji}}$ é o custo que $\mathrm{o}$ arbitrador deve incidir para transportar o produto do mercado $j$ para o mercado $i$. 


\subsection{Modelo analítico}

Dado que os preços $\mathrm{P}_{\mathrm{i}}$ e $\mathrm{P}_{\mathrm{j}}$ são determinados simultaneamente em mercados integrados, tem-se que Pj não é independente de u, e a aplicação dos Mínimos Quadrados Ordinários para a estimação da elasticidade de transmissão de preços (parâmetro $\beta$ em (1)) provocaria estimativas viesadas e inconsistentes. Além do mais, comumente, as séries de preços $\mathrm{P}_{\mathrm{i}}$ e $\mathrm{P}_{\mathrm{i}}$ não são estacionárias.

Para uma série ser estacionária, esta deve seguir as seguintes pressuposições: $E(y t)=\mu$ (média constante), $\operatorname{var}(y t)=\sigma 2$ (variância constante) e $\operatorname{cov}(y t, y t+s)=\operatorname{cov}(y t, y t-s)$ (covariância depende de $s$ e não de $t)$, caso contrário, a série é não-estacionária. A realização de regressão com séries não estacionárias pode acarretar em estimadores de mínimos quadrados e testes estatísticos errôneos.

O método mais utilizado para detectar a estacionariedade de uma série temporal é o teste de raiz unitária. De acordo com Harris e Sollis (2003) para testar a presença de raiz unitária pode-se utilizar o teste Dickey-Fuller Aumentado (ADF) e o teste de Phillips e Perron (PP), nos quais testam a hipótese nula de que as séries apresentam raiz unitária, ou seja, são não estacionárias.

Além da necessidade de realização dos testes de estacionariedade, Hatemi-J e Irandoust (2005) indicam a realização de testes de co-integração. Engle e Granger (1987), apontam que co-integração é uma combinação linear de variáveis não-estacionárias e integradas de mesma ordem, que no longo prazo, compartilham uma relação de equilíbrio. Os métodos mais utilizados para determinação das relações de co-integração entre as variáveis são os de Engle e Granger e de Johansen. O método utilizado neste trabalho é o de Johansen (1988), pois ao contrário do de Engle e Granger, este permite a estimação dos parâmetros da relação de equilíbrio entre variáveis não estacionárias e a presença de múltiplos vetores de co-integração.

Johansen (1988) propõe os testes de razão de verossimilhança traço e máximo autovalor para identificar o número de vetores de co-integração. $\mathrm{O}$ teste do traço examina a hipótese de que existem, no máximo, $r$ vetores de co-integração $\left(\mathrm{H}_{0}=\mathrm{r} \leq \mathrm{r}_{0}\right.$, contra $\left.\mathrm{H}_{1}=\mathrm{r}>\mathrm{r}_{0}\right)$, enquanto que no teste de máximo autovalor, a hipótese nula de que há $r$ vetores de co-integração, contra a hipótese alternativa de que há $(r+1)$.

A integração de preços da soja entre os estados do Mato Grosso, do Paraná e do Rio Grande do Sul será baseada modelo de autorregressão vetorial (VAR).

Conforme Gujarati e Porter (2011) o VAR desenvolvido por Sims (1980) tem como objetivo analisar as relações lineares entre cada variável e seus valores defasados, considerando os problemas básicos, como a conjugação de variáveis que possam melhor explicar o problema de pesquisa, assim como o total de defasagens arroladas às variáveis do modelo. O VAR pode ser representado da seguinte maneira:

$$
\beta \mathrm{X}_{\mathrm{t}}=\beta_{0}+\sum_{\mathrm{j}=1}^{\mathrm{K}} \beta_{\mathrm{i}} \mathrm{X}_{\mathrm{t}-\mathrm{i}}+\mathrm{u}_{\mathrm{t}}
$$


em que $\mathrm{X}_{\mathrm{t}}$ é um vetor representado por uma matriz $(n \times 1)$; $\beta_{0}$ é um parâmetro que pode assumir os seguintes valores: $\mathrm{i}=0,1,2,3, \ldots, \mathrm{k}$, matricialmente representado por: $(n \times n)$; e ut representa os termos de erros com vetor $(n \times 1)$, que na linguagem do VAR são chamados de impulsos, inovações ou choques.

A identificação das relações entre as variáveis do modelo tanto no curto como no longo prazo, são realizadas pelos Vetores de Correção de Erro (VEC) é um VAR (reparametrizado) com as restrições de co-integração entre as variáveis. O VEC pode ser representado da seguinte forma (LIMA, 2014):

$$
\Delta \mathrm{Y}_{\mathrm{t}}=\Gamma_{1} \Delta \mathrm{Y}_{\mathrm{t}-1}+\Gamma_{2} \Delta \mathrm{Y}_{\mathrm{t}-2}+\ldots+\Gamma_{\mathrm{p}-1} \Delta \mathrm{Y}_{\mathrm{t}-(\mathrm{p}-1)}+\alpha \beta^{\prime} \mathrm{Y}_{\mathrm{t}-1}+\mathrm{u}_{\mathrm{t}}
$$

em que são as $r$ relações de co-integração que definem a trajetória de longo prazo (equilíbrio) entre as variáveis; $\alpha$ é a matriz de coeficientes de ajustamento para o equilíbrio de longo prazo e $\Gamma_{\mathrm{i}}$ matrizes de coeficientes que definem a dinâmica de curto prazo.

Lima (2014) evidencia que testes nos coeficientes das matrizes $\alpha$ e $\beta$ são usados para análise de hipóteses sobre as características de integração dos mercados, bem como indicam variáveis que podem ser consideradas (fracamente) exógenas na análise. Podem, também, indicar quais mercados fazem parte do equilíbrio de longo prazo e se a integração entre os mercados é perfeita dando suporte à Lei do Preço Único. Ainda conforme o autor, no teste em $\beta$, o teste de $t$ de Student indica a significância das variáveis no vetor de co-integração não necessitando a realização do teste de Razão de Verossimilhança. Já o teste em $\alpha$, indica quais variáveis podem ser consideradas fracamente exógenas no mercado. Caso uma variável seja considerada exógena, sua equação não precisa ser estimada. Ela afeta as outras, mas não é afetada. Ao mesmo tempo, pode-se testar a hipótese de igualdade dos coeficientes de ajustamento de diferentes variáveis. Assim, podem-se testar quais mercados fazem parte do equilíbrio de longo prazo e se a integração entre eles é perfeita confirmando a LPU.

De acordo com Lima (2014), para se ter o conhecimento da causalidade (precedência) de uma variável em outra, é necessário a realização do teste de causalidade de Granger. $\mathrm{O}$ teste estima as seguintes equações:

$$
\begin{aligned}
& Y_{1}=\alpha_{0}+\sum_{j=1}^{k} \beta_{Y i} Y_{t-i}+\sum_{j=1}^{k} \beta_{X i} X_{t-i}+u_{1 t} \\
& X_{1}=\gamma_{0}+\sum_{j=1}^{k} \beta_{Y i} X_{t-i}+\sum_{j=1}^{k} \beta_{\gamma i} Y_{t-i}+u_{2 t}
\end{aligned}
$$

em que $Y_{t}$ é o preço da soja na região $Y$; $Y_{t-1}$ é o preço da soja na região $Y$ defasado em $i$ períodos; $X_{t}$ é o preço da soja na região $\mathrm{X} ; X_{t-1}$ é o preço da soja na região $X$ defasado em $i$ períodos; $\mathrm{u}_{1 \mathrm{t}}$ e $\mathrm{U}_{2 \mathrm{t}}$ são as perturbações, as quais supõe-se que não tenham correlações. $\mathrm{O}$ autor discorre sobre quatro possíveis situações: (a) causali- 
dade unidirecional de para $X$ para $Y$; (b) causalidade unidirecional de $Y$ para $X$; (c) causalidade bidirecional de $X$ para $Y$ e de $Y$ para $X$; e (d) ausência de causalidade.

\subsection{Dados}

Os dados utilizados neste trabalho referem-se às séries mensais médias de preços, em US\$, da soja em grão, tipo exportação, obtidas do Centro de Estudos Avançados em Economia Aplicada (CEPEA) da ESALQ / USP, considerando o período de janeiro de 2005 a dezembro de 2015.

Foram consideradas três séries de preços referentes aos três maiores estados produtores de soja do Brasil: Mato Grosso ((LN_MT), Paraná (LN_PR) e Rio Grande do Sul (LN_RS). Para representar o preço da soja de cada um dos estados, tomou-se o preço colhido pelo município de Sorriso como representativo do Mato Grosso. No Paraná, a média mensal da praça do sudoeste do Paraná, oeste do Paraná, norte do Paraná, Ponta Grossa e Paranaguá. Enquanto no Rio Grande do Sul, tomou-se a média mensal dos preços de Passo Fundo e Ijuí. A escolha dessas regiões foi fundamentada na disponibilidade dos dados. As séries de preços utilizadas são em dólares americanos e transformadas em logaritmos, de forma que os coeficientes betas obtidos correspondessem às elasticidades de transmissão de preços. Quanto ao software, foi utilizado o Eviews versão 8.

\section{Resultados e discussão}

Nesta seção, serão apresentados e discutidos os resultados da presente pesquisa. Conforme explicitado na seção de metodologia, as séries temporais utilizadas precisam ser estacionárias. Dessa forma, o primeiro procedimento adotado contempla a realização do teste de raiz unitária denominada Dickey-Fuller Aumentado (ADF) e do Phillips-Perron (PP). Estimaram-se três modelos: (I) sem tendência e com intercepto; (II) com tendência e com intercepto; e (III) sem tendência e sem intercepto. Todos os casos utilizam os logaritmos das séries de preços futuros da soja, para cada um dos estados utilizados na análise. Os modelos foram estimados primeiramente em nível e depois em primeira diferença. Assim, os resultados dos testes de estacionariedade das variáveis podem ser verificados na Tabela 1. 
Tabela1 - Resultado dos testes ADF e PP em nível e em primeira diferença para as séries mensais de soja nos estados de Mato Grosso (LN_MT, Paraná (LN_PR) Rio Grande do Sul (LN_RS), janeiro de 2005 a dezembro de 2015

\begin{tabular}{cccccccc}
\hline $\begin{array}{c}\text { Séries em } \\
\text { Nível }\end{array}$ & I & II & III & $\begin{array}{c}\text { Séries em } \\
\text { primeira } \\
\text { diferença }\end{array}$ & I & II & III \\
\hline \multicolumn{7}{c}{ DickerFuller Aumentado (ADF)* } \\
\hline LN_MT & $-2,25$ & $-1,71$ & 0,32 & LN_MT & $-9,09$ & $-9,24$ & $-9,10$ \\
LN_PR & $-2,25$ & $-1,69$ & 0,36 & LN_PR & $-9,43$ & $-\mathbf{9 , 5 8}$ & $-9,43$ \\
LN_RS & $-2,53$ & $-2,11$ & 0,30 & LN_RS & $-8,79$ & $-8,94$ & $-8,80$ \\
\hline & $-2,06$ & $-1,39$ & 0,34 & LN_MT & $-9,12$ & $-9,27$ & $-9,13$ \\
\hline LN_MT & $-2,02$ & $-1,50$ & 0,31 & LN_PR & $-9,47$ & $-9,59$ & $-9,47$ \\
LN_PR & $-2,13$ & $-1,65$ & 0,25 & LN_RS & $-8,82$ & $-8,97$ & $-8,83$ \\
LN_RS & $-2,13$ & Philips-Perron (PP)* \\
\hline
\end{tabular}

Fonte: elaboração própria com base nos resultados da pesquisa.

Nota: Valores significativos a $1 \%$ em negrito.

*Os valores críticos ao nível de significância de 1\% são -3,148; -4,030; e -2,583, respectivamente, nos modelos I, II e III.

**Modelo I é com intercepto, modelo II com tendência e intercepto e modelo III sem tendência e intercepto

Como pode ser observado, ao se considerar os logaritmos das séries de preços da soja em nível, a hipótese nula de que existe raiz unitária não pôde ser rejeitada. Logo, as séries de preços da soja são não estacionárias, assim como encontrado por Vieira (2014), ao considerar os preços da soja do Rio Grande do Sul e da bolsa de Chicago. Com base em tais resultados, repetiu-se o ajustamento para avaliar se as séries tornam-se estacionárias quando analisadas em primeira diferença. Esses resultados, por sua vez, mostram-se significativos, indicando que as variáveis em questão são integradas de ordem um $[\mathrm{I}(1)]$, sendo esse o primeiro passo para a identificação de uma relação de equilíbrio de longo prazo entre as séries analisadas, atendendo, assim, as condições iniciais para a estimação do modelo vetorial de correção de erros (VEC).

Após a identificação da ordem de integração, verifica-se o número de defasagens que devem ser utilizadas no modelo auxiliar VAR, necessário para a posterior estimação do VEC. Na Tabela 2, tais resultados podem ser vistos, tendo como base os critérios do Estimador de Máxima Verossimilhança (LR), Erro de Previsão Final (FPE), Akaike (AIC), Schwarz (SC) e Hannan-Quinn (HQ).

Com base nas informações da Tabela 2, percebe-se que por todos os critérios utilizados, com exceção do Schwarz (SC), o modelo deve conter duas defasagens, 
quando se relaciona a série de preços da soja entre os estados do Mato Grosso, Rio Grande do Sul e Paraná. Deste modo, opta-se por incluir duas defasagens no modelo, dado que foi indicado pela maioria dos testes (LR, FPE, AIC e HQ).

Tabela 2 - Determinação do número de defasagens no modelo VAR para as séries de preço da soja dos estados considerados na análise, janeiro de 2005 a dezembro de 2015

\begin{tabular}{cccccc}
\hline Lag & LR & FPE & AIC & SC & HQ \\
\hline 0 & - & $1,92 \mathrm{E}-07$ & $-6,95129$ & $-6,88306$ & $-6,92357$ \\
1 & 498,7003 & $3,48 \mathrm{E}-09$ & $-10,962$ & $-10,68904 *$ & $-10,8511$ \\
2 & $39,79833^{*}$ & $2,87 \mathrm{e}-09 *$ & $-11,15696 *$ & $-10,6793$ & $-10,96294 *$ \\
\hline
\end{tabular}

Fonte: Elaboração própria com base nos resultados da pesquisa.

Nota: * Indica a ordem selecionada pelo critério.

LR: Estimador de máxima verossimilhança

FPE: Erro de previsão final

AIC: Critério de informação de Akaike

SC: Critério de informação de Schwarz

HQ: Critério de informação de Hannan-Quinn

O próximo passo é a realização do teste de co-integração de Johansen, com o intuito de identificar o número de relações de equilíbrio de longo prazo entre os três mercados de soja analisados (Tabela 3).

Tabela 3 - Resultados do teste de co-integração de Johansen para as séries de preços mensais de soja exportada pelos estados considerados na análise, janeiro de 2005 a dezembro de 2015

\begin{tabular}{ccccc}
\hline Hipótese & Teste do & Valor Crítico & Teste do Máximo & Valor Crítico (5\%) \\
Nula & Traço & $(5 \%)$ & Autovalor & \\
$\mathrm{r}=0 *$ & 65,00719 & 42,91525 & 35,02653 & 25,82321 \\
$\mathrm{r}<1 *$ & 29,98065 & 25,87211 & 24,71925 & 19,38704 \\
$\mathrm{r}<2$ & 5,261409 & 12,51798 & 5,261409 & 12,51798 \\
\hline
\end{tabular}

Fonte: Elaboração própria com base nos resultados da pesquisa.

Nota: *Indica rejeição da hipótese nula a 5\% de significância.

Os resultados do teste do traço e do máximo autovalor, encontrados na Tabela 3, indicam rejeição da hipótese nula de que não há vetor de co-integração e re- 
jeição da hipótese da existência de um vetor de co-integração entre as séries relacionadas dos estados do Mato Grosso, Rio Grande do Sul e Paraná, considerando-se o nível de 5\% de significância. Além disso, tem-se que a hipótese nula da existência de no máximo duas relações de co-integração não pode ser rejeitada, pois a estatística apresentada é menor do que seu respectivo valor crítico. Dessa forma, como as hipóteses alternativas não foram rejeitadas, pode-se dizer que as séries temporais de preços de soja são co-integradas, havendo um equilíbrio de longo prazo entre os estados, sendo identificadas ainda duas relações de co-integração. Souza e Campos (2009) também encontram relação de equilíbrio de longo prazo entre o preço da soja nestes mesmos estados, considerando o intervalo de janeiro de 2001 a fevereiro de 2008.

Assim, as relações de longo prazo entre os preços de soja dos estados considerados são representadas por duas equações de co-integração, sendo a (8) referente a relação entre os preços do Mato Grosso e Paraná, e a (9) referente a relação entre Rio Grande do Sul e Paraná.

\begin{tabular}{|c|c|c|}
\hline \multicolumn{2}{|c|}{ LN_MT $=-0,53+0,89$ LN_PR } & \multirow{2}{*}{} \\
\cline { 1 - 2 } Erro Padrão & $(-0,02)$ & \\
\hline Estatística T & $-44,39$ & \\
\hline & & \\
\hline \multicolumn{2}{|c|}{ LN_RS $=-0,15+0,95$ LN_PR } & \\
\hline Erro Padrão & $(-0,02)$ & \\
\hline Estatística T & $-47,99$ & \\
\hline
\end{tabular}

De acordo com as equações estimadas, verifica-se que o coeficiente da variável representativa do Paraná é estatisticamente significativo a 1\%. Ademais, pelo fato das variáveis terem sido transformadas em seu logaritmo natural, os resultados devem ser interpretados como elasticidades, isto é, como uma medida da reposta de longo prazo, em termos percentuais, da variável dependente em relação a mudanças percentuais nas demais variáveis. Tais coeficientes representam as elasticidades de transmissão de preços (parâmetro $\beta$ em (1)). Deste modo, a elasticidade encontrada para o preço da soja no Paraná na equação (8) indica que um aumento de $10 \%$ no preço da soja nesse estado estaria associado a uma elevação do preço desta commodity no estado do Mato Grosso em 8,9\%. De forma semelhante, Souza e Campos (2009) também identificam uma elasticidade de transmissão de preço próximo à unidade $(9,8 \%)$, considerando a relação desses estados. Quanto à equação (9), a elasticidade estimada para o preço da soja no Paraná indica que uma elevação de $10 \%$ em seu preço, aumentando o preço do produto no Rio Grande do Sul em 9,5\%.

As equações analisadas anteriormente deram suporte para identificar a relação de equilíbrio de longo prazo entre as variáveis analisadas. No entanto, desequilí- 
brios podem ocorrer no curto prazo, devendo haver algum mecanismo que conduza as variáveis para o equilíbrio. O mecanismo supracitado é obtido pelo modelo VEC por meio dos coeficientes de correção de erro, os quais podem ser interpretados como a correção percentual por período de um desequilíbrio de curto prazo. Os coeficientes de ajustamento para cada uma das equações de co-integração estimadas são apresentados na Tabela 4.

Tabela 4 - Coeficientes de correção de erro do modelo VEC para as para as séries LN_MT, LN_RS, LN_PR

\begin{tabular}{cccc}
\hline & $\Delta$ LN_MT & $\Delta$ LN_RS & $\Delta$ LN_PR \\
\hline Relação de Co-Integração 1 & \\
\hline Coeficiente & 0,42118 & 0,11364 & 0,0613 \\
Erro padrão & $-0,17382$ & $-0,20047$ & $-0,18674$ \\
Estat. T & $-2,42309 * * *$ & $-0,56687^{\text {NS }}$ & $-0,32827^{\text {NS }}$ \\
\hline & Relação de Co-Integração 2 & \\
\hline Coeficiente & $-0,027966$ & 0,36501 & $-0,049737$ \\
Erro padrão & $-0,16386$ & $-0,18898$ & $-0,17604$ \\
Estat. T & 0,17067 & $-1,93142 * * *$ & 0,28253 \\
\hline
\end{tabular}

Fonte: Resultados da pesquisa.

Nota: *** Significativo a $1 \%$, NS não significativo.

De acordo com a Tabela 4, os coeficientes de ajustamento $(\alpha)$ mostram como as variáveis reagem a desequilíbrios dos preços no curto prazo. Em relação à co-integração 1, apenas os preços do Mato Grosso são estatisticamente significativos a $1 \%$, indicando que $42,12 \%$ do desequilíbrio de curto prazo referente à trajetória de longo prazo do preço da soja do Estado são corrigidos mensalmente, ou seja, seriam necessários em média, 2,4 meses para que o desequilíbrio fosse sanado. No tocante à co-integração 2, apenas os preços do Rio Grande do Sul são estatisticamente significativos a $1 \%$, mostrando que $36,50 \%$ do desequilíbrio do preço da commodity no curto prazo é corrigido por mês, indicando que seriam necessários 2,7 meses para a correção total no preço da soja neste mercado.

Adicionalmente, busca-se examinar os efeitos de causalidade (precedência) estatística de curto prazo entre os estados de forma a identificar se as alterações em um dado mercado antecedem, de forma ordenada, às alterações em outro mercado. Para essa análise, é realizado o teste de causalidade de Granger com o uso das séries com duas defasagens ${ }^{4}$ (Tabela 5).

4 Ressalta-se que, para a estimação do teste de causalidade de Granger, o número de defasagens foi 
Tabela 5 - Resultados do teste de Granger para as séries de preços mensais de soja exportada pelos estados considerados na análise, janeiro de 2005 a dezembro de 2015

\begin{tabular}{ccc}
\hline Hipóteses nulas & Probabilidade & Resultado \\
\hline LN_PR não causa LN_RS & 0,31 & Não rejeito \\
LN_RS não causa LN_PR & 0,00 & Rejeito \\
LN_MT não causa LN_RS & 0,84 & Não rejeito \\
LN_RS não causa LN_MT & 0,13 & Não rejeito \\
LN_MT não causa LN_PR & 0,10 & Rejeito \\
LN_PR não causa LN_MT & 0,07 & Rejeito \\
\hline
\end{tabular}

Fonte: Elaboração própria com base nos resultados da pesquisa.

Nota-se com os resultados da Tabela 5 que, considerando-se um nível de 1\% de significância, apenas uma hipótese nula é rejeitada, ou seja, percebe-se que o preço do Paraná é causado (precedido) pelo preço do Rio Grande do Sul, bem como é causado pelo preço do Mato Grosso ao nível de 10\%. Esse resultado dá indícios da variabilidade do preço da soja no Paraná como resultado de choques nos preços dos demais estados estudados. Ainda considerando-se o nível de $10 \%$ de significância, nota-se que o preço do Mato Grosso também é causado pelo preço do Paraná, assim, isso indica uma causalidade, segundo Granger, bidirecional, ou seja, os preços defasados de uma localidade ajudam na previsão do preço da outra localidade, e vice-versa. As demais variáveis de preços não precedem umas às outras, temporalmente, no sentido de Granger, pois não rejeitam a hipótese nula de ausência de causalidade. Esse resultado mostra que qualquer choque em um desses estados pode não afetar, no curto prazo, a outro.

São estimadas ainda as funções impulso resposta com objetivo de analisar o comportamento dos preços de soja no Mato Grosso, Paraná e Rio Grande do Sul em até 24 meses após um choque exógeno nessas variáveis, como é demonstrado nas Figuras 1, 2 e 3. A Figura 1 mostra que um choque de um desvio padrão no preço da soja no Rio Grande do Sul afeta positivamente os preços no Mato Grosso atingindo seu ápice no quarto mês, decrescendo até décimo primeiro mês onde se estabiliza acima do preço inicial. Enquanto que um choque no Paraná eleva os preços no Mato Grosso até o oitavo mês, onde se estabiliza a um preço superior ao inicial.

escolhido com base nos mesmos critérios aplicados para a estimação do modelo VAR, apresentados na Tabela 2. 
Figura 1 - Função Impulso resposta do preço da soja no Mato Grosso a choques nos preços do Rio Grande do Sul e Paraná.

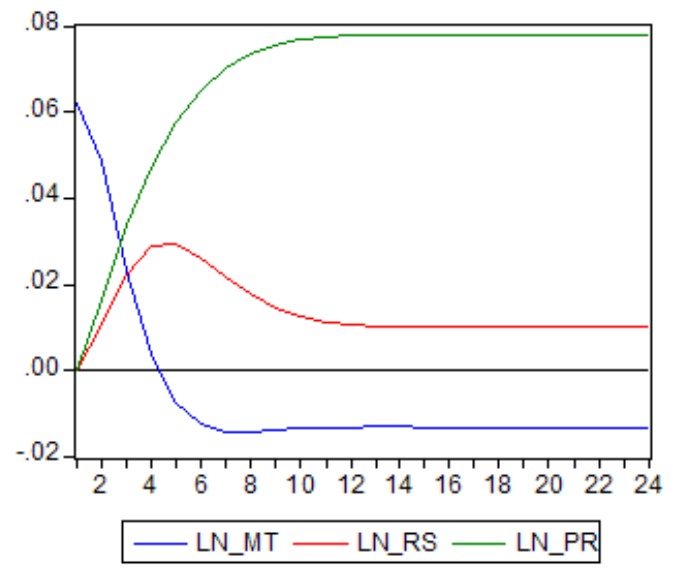

Fonte: Resultado da pesquisa.

Em relação à resposta do preço da soja no Paraná a um choque no Rio Grande do Sul, verifica-se que esse se eleva até o segundo mês, o qual decresce até o nono mês estabilizando-se acima do nível inicial de preço. Quanto ao efeito do choque no preço do Mato Grosso, esse decresce até o terceiro mês, onde se estabiliza abaixo do preço inicial, evidenciando uma resposta negativa dos preços no Paraná a tais choques.

Figura 2 - Função Impulso resposta do preço da soja no Paraná a choques nos preços do Rio Grande do Sul e Mato Grosso.

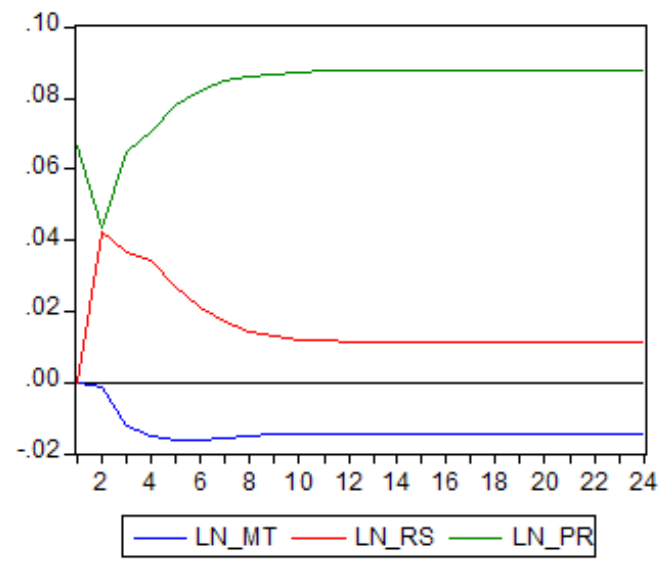

Fonte: Resultado da pesquisa. 
No que tange a resposta do preço da soja no Rio Grande do Sul a choques no Paraná, verifica-se uma elevação dessa até o sexto mês, onde se estabiliza acima do preço inicial. Já para o Mato Grosso, um choque no preço nesse estado afeta negativamente o preço no Paraná até o quarto mês, posteriormente elevando o preço até o oitavo, onde se estabiliza abaixo do nível inicial de preço.

Figura 3 - Função Impulso resposta do preço da soja no Rio Grande do Sul a choques nos preços do Paraná e Mato Grosso.

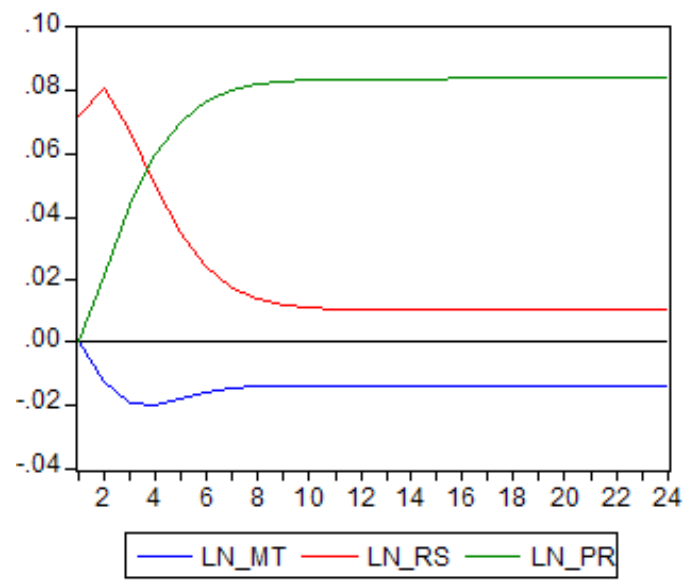

Fonte: Resultado da pesquisa.

Diferentemente de Souza e Campos (2009), os desequilíbrios causados pelos choques não antecipados nos preços da soja dos diferentes estados permanecem estáveis em níveis diferentes dos iniciais. Além disso, verifica-se que choques no preço da soja no Mato Grosso geram reduções nos preços iniciais do Paraná e do Rio Grande do Sul, o que pode estar relacionado com o fato desse estado ser o principal produtor de soja do País, correspondendo a uma produção equivalente à dos outros dois estados juntos, segundo dados da CONAB (2016). Nesse sentido, choques econômicos não esperados em tal mercado de referência podem influenciar negativamente os níveis de preços dos demais.

A Tabela 6 apresenta os resultados da decomposição da variância dos erros de previsão dos preços do Mato Grosso, Paraná e Rio Grande do Sul, no período entre janeiro de 2005 a dezembro de 2015. Em relação aos resultados para o preço do Mato Grosso (LN_MT), verifica-se que, após 12 meses de um choque não antecipado, 83,1\% da variância do erro de previsão deve-se à influência do próprio preço, sendo o restante atribuído aos preços dos outros estados. Similarmente, as variâncias dos erros de previsão do Paraná (LN_PR) e do Rio Grande do Sul (LN_RS) também são influenciadas majoritariamente por LN_MT, que explicam, respectivamente, $78,6 \%$ e 78,8\% da variância. Nesta mesma direção, Souza e Campos (2009) 
também identificam os preços do Mato Grosso como sendo o principal responsável pela variância dos erros de previsão nos demais estados analisados.

Tabela 6 - Decomposição da variância dos erros de previsão de LN_MT, LN_PR e LN_RS.

\begin{tabular}{cccccccccc}
\hline \multirow{2}{*}{ Período } & $\begin{array}{c}\text { Decomposição da variância } \\
\text { dos erros de previsão de }\end{array}$ & \multicolumn{2}{c}{$\begin{array}{c}\text { Decomposição da variância } \\
\text { dos erros de previsão de }\end{array}$} & \multicolumn{3}{c}{$\begin{array}{c}\text { Decomposição da variância } \\
\text { dos erros de previsão de }\end{array}$} \\
& \multicolumn{3}{c}{ LN_MT } & \multicolumn{3}{c}{ LN_PR } & \multicolumn{3}{c}{ LN_RS } \\
& LN_MT & LN_PR & LN_RS & LN_MT & LN_PR & LN_RS & LN_MT & LN_PR & LN_RS \\
1 & 100 & 0 & 0 & 79,965 & 16,637 & 3,399 & 83,704 & 0 & 16,296 \\
3 & 96,586 & 1,539 & 1,875 & 82,848 & 9,243 & 7,909 & 82,119 & 1,852 & 16,029 \\
6 & 89,296 & 5,568 & 5,136 & 80,689 & 11,206 & 8,113 & 80,639 & 6,343 & 13,018 \\
9 & 85,282 & 8,833 & 5,884 & 79,387 & 13,078 & 7,535 & 79,547 & 9,505 & 10,948 \\
12 & 83,099 & 10,940 & 5,962 & 78,651 & 14,201 & 7,148 & 78,838 & 11,390 & 9,773 \\
\hline
\end{tabular}

Fonte: Elaboração própria com base nos resultados da pesquisa.

Por fim, para a verificação da LPU nesses mercados, é necessário impor algumas restrições, como explicita Souza e Campos (2009). Para Lima (2014) para realização de uma análise de integração de mercados, os testes nos coeficientes das matrizes alfa $(\alpha)$ e beta $(\beta)$ são usados para análise de hipóteses sobre as características de integração dos mercados. Tais testes podem sugerir quais mercados fazem parte do equilíbrio de longo prazo e se a integração entre eles é perfeita dando suporte à LPU.

Tabela 7 - Teste de significância de restrição sobre o parâmetro de longo prazo $(\beta)$ do vetor de co-integração estimado

\begin{tabular}{cc}
\hline Hipótese & Razão de verossimilhança \\
$\beta(1,1)=0$ & $6,59^{*}$ \\
$\beta(1,2)=0$ & $10,02^{*}$ \\
$\beta(1,3)=0$ & $0,29^{\mathrm{NS}}$ \\
\hline$\beta(1,1)-\beta(1,2)-\beta(1,3)=0$ & $6,88^{*}$ \\
\hline
\end{tabular}

Fonte: Elaboração própria com base nos resultados da pesquisa.

Nota: * Significância a 5\%; NS: não significante.

Os resultados apresentados na Tabela 7 mostram a rejeição da hipótese nula de $\beta i=0$ para as séries de preços do Mato Grosso e Rio Grande do Sul, o que significa que tais regiões participam das relações de co-integração e exercem influência nos movimentos dos preços do mercado de soja. Percebe-se ainda a rejeição da hipótese de perfeita integração no mercado pela significância da relação 
$\beta(1,1)-\beta(1,2)-\beta(1,3)=0$, indicando diferenças nos vetores de co-integração.

Tabela 8 - Teste de significância de restrição sobre o parâmetro de curto prazo $(\alpha)$ do vetor de co-integração estimado

\begin{tabular}{cc}
\hline Hipótese & Razão de verossimilhança \\
$\alpha(1,1)=0$ & $1,35^{\mathrm{NS}}$ \\
$\alpha(2,1)=0$ & $0,94^{\mathrm{NS}}$ \\
$\alpha(3,1)=0$ & $0,13^{\mathrm{NS}}$ \\
\hline$\alpha(1,1)-\alpha(2,1)-\alpha(3,1)=0$ & $2,83^{\mathrm{NS}}$ \\
\hline
\end{tabular}

Fonte: Elaboração própria com base nos resultados da pesquisa.

Nota: *Significância a 5\%; NS: não significante.

$\mathrm{Na}$ Tabela 8, têm-se os resultados dos testes sobre os parâmetros alfa $(\alpha)$, que determina como cada variável reage e se ajusta a um desequilíbrio de curto prazo, de modo que regresse ao padrão de equilíbrio de longo prazo (coeficientes de correção de erro (Tabela 4)). Os resultados apontam rejeição da hipótese nula para todos os vetores analisados, evidenciando uma possível integração perfeita nos mercados de soja analisados, indo de encontro com os resultados das estimativas das equações (8) e (9), os quais mostraram que variações nos preços do Paraná eram transmitidas quase integralmente (parâmetros próximos à unidade) para o Mato Grosso e Rio Grande do Sul.

\section{Considerações Finais}

Nesse trabalho, buscou-se investigar a integração de preços da soja exportada pelos estados de Mato Grosso, Paraná e Rio Grande do Sul, sob a observação de validade da Lei de Preço Único. A justificativa para tal investigação se dá pela necessidade de compreensão do processo de transmissão de preço da principal commodity exportada pelo Brasil, uma vez que essa pode vir a facilitar formulação de políticas de comercialização adequadas ao desenvolvimento do setor, podendo auxiliar na determinação da amplitude dos efeitos dessas políticas em uma região sobre as demais.

Foi realizado o teste de raiz unitária de Dickey e Fuller e Phillips-Perron, de co-integração de Johansen, causalidade de Granger, análise das funções impulso resposta, análise da decomposição da variância e análises no vetor de co-integração, bem como por meio da imposição sobre parâmetros $\beta$ e $\alpha$.

Os resultados obtidos para a análise do mercado de soja brasileiro evidenciaram que os preços do produto nos principais mercados são co-integrados, ou seja, apresentaram uma relação de equilíbrio no longo prazo. De modo geral, verificou-se que variações no preço da soja no Paraná são transmitidas quase integralmente 
para o Mato Grosso e Rio Grande do Sul. Os coeficientes de correção de erro ainda apontaram que os preços da soja se ajustam rapidamente a desequilíbrios de curto prazo nos preços nacionais desta commodity. Os resultados das funções impulso resposta corroboraram para tais indícios de rápida recuperação e estabilização dos preços.

Além disso, com base nos vetores de co-integração e no teste do coeficiente alfa, foi identificado mercados perfeitamente integrados, ou seja, houve validação da Lei de Preço Único no mercado de soja exportada.

Por fim, percebeu-se a importância de cada estado na formação do preço da soja exportada, já que eles respondem de forma rápida às variações de preços. Para futuras pesquisas, torna-se relevante analisar como o preço internacional desta commodity influencia o equilíbrio do mercado interno de soja.

\section{Referências}

COMPANHIA NACIONAL DE ABASTECIMENTO - CONAB. Disponível em: <www.conab.gov.br>. Acesso em 15 de abril de 2016.

CUNHA, Dênis Antônio da. Integração de preços no mercado internacional de café. Dissertação de Mestrado em Economia. Universidade Federal de Viçosa - Viçosa, MG, 2008. 105 p.

CUNHA, D. A., LIMA, J. E.; BRAGA, M. J. Integração espacial do mercado de boi

gordo: uma na análise de cointegracao com threshold. Análise Econômica, ano 28, n. 53, p.251-67, 2010.

CHRISTOFOLETTI, Maria Alice Móz; SILVA, Rodolfo Margato; MARTINES-FILHO, João Gomes. Cointegração e Causalidade no Mercado de Soja:

Análises para Brasil, China e EUÁ. Conferência em Gestão de Risco e Comercialização de commodities. BM\&F Bovespa, 2011.

FACKLER, P.; GOODWIN, B.K..Spatial price analysis: a methological review. North Carolina, Department of Agricultural and Resourse Economics.North Carolina State University, 2000.

FREITAS, C. O. de ; LÍRIO, V. S.; CORONEL, D. A. Análise da transmissão de preço do fumo entre os estados produtores brasileiros e no mercado internacional. Revista Ciências Administrativas, v. 18, n. 1, 2014.

GOODWIN, B. K.; PIGOTT, N. E. Spatial market integration in the presence of threshold effects. American Journal of Agriculture Economics, v. 83, n. 2, p. 302317,2001 . crossref https://doi.org/10.1111/0002-9092.00157

GUJARATI, D. N.; PORTER, D. C. Econometria Básica, $5^{a}$ ed. Bookman: Porto Alegre, 2011. 
HATEMI-J, A.; IRANDOUST, M. Bilateral trade elasticities: Sweden versus her trade partners. American Review of Political Economy, vol. 3, nº 2. 2005.

HARRIS, R.; SOLLIS, R. Applied Time Series Modeling and Forecasting. England: John Wiley \& Sons, 2003, p. 302.

JOHANSEN, S. Statistical Analysis of Cointegration Vectors. Journal of Economic Dynamics and Control, v. 12, n. 1, p. 231-254, 1988. crossref https://doi. org/10.1016/0165-1889(88)90041-3

KRUGMAN, P. R.; OBSTFELD, M. International economics: theory and policy. 5. ed. Massachusetts:AddsonWelsley, 2005.

LIMA, J. E de. Modelos Multivariados de Séries Temporais. DER-UFV, 2014.

LOVADINE, Débora. Causalidade e transmissão de preços entre mercado interno $e$ internacional para produtos do Complexo da soja. 2004. Monografia de Ciências Econômicas. Universidade de São Paulo, USP, Brasil.

MATTOS, Leonardo Bornacki. Efeitos de custo de transação sobre a integração espacial de mercado regionais de carne de frango no Brasil. Tese de Doutorado em Economia Aplicada. Universidade Federal de Viçosa - Viçosa, MG, 2008. 162 p.

SILVA, Wesley Vieira da; SANTO, Elinaldo Leal; SILVA, LSC. Co-integração entre os preços da soja cotados nos mercados brasileiro e norte-americano: uma análise empírica. Caderno de Pesquisas em Administração. São Paulo, v. 10, n. 3, p. 69-78, 2003.

SOUSA, E. P.; CAMPOS, A. C. Transmissão inter-regional de preços no mercado brasileiro de soja. Revista Análise Econômica, Porto Alegre, ano 27, n. 51, p.83-105, março de 2009.

TARDELLI, B. L. S.. Integração espacial da soja escoada do Mato Grosso para o porto de Santos: uma análise sob custos de transação. Anais...XIEncontro Nacional de Economia (ANPEC),Porto de Galinhas - PE. 2012.

VIEIRA, A. C. K. Análise da transmissão de preço da soja entre mercado físico e mercado futuro, com base nas cooperativas Cotrirosa e Coopatrigo, e na bolsa Chicago, no período entre 2003 a 2013. Revista da Graduação, v. 8, n. 1, 2015.

Recebido em 03.11.16

Aprovado em 19.06.17 
